\title{
RNA-seq analysis of gene expression changes during pupariation in Bactrocera dorsalis (Hendel) (Diptera: Tephritidae)
}

\author{
Er-Hu Chen ${ }^{1 \dagger}$, Qiu-Li Hou ${ }^{1 \dagger}$, Wei Dou ${ }^{1,2+}{ }^{+}$, Dan-Dan Wei ${ }^{1,2}$, Yong Yue ${ }^{1}$, Rui-Lin Yang ${ }^{1}$, Shuai-Feng Yu', \\ Kristof De Schutter ${ }^{3}$, Guy Smagghe ${ }^{1,2,3^{*}}$ and Jin-Jun Wang ${ }^{1,2^{*}}$ (D)
}

\begin{abstract}
Background: The oriental fruit fly, Bactrocera dorsalis (Hendel) has been considered to be one of the most important agricultural pest around the world. As a holometabolous insect, larvae must go through a metamorphosis process with dramatic morphological and structural changes to complete their development. To better understand the molecular mechanisms of these changes, RNA-seq of B. dorsalis from wandering stage (WS), late wandering stage (LWS) and white puparium stage (WPS) were performed.

Results: In total, 11,721 transcripts were obtained, out of which 1914 genes (578 up-regulated and 1336 downregulated) and 2047 genes (655 up-regulated and 1392 down-regulated) were found to be differentially expressed between WS and LWS, as well as between WS and WPS, respectively. Of these DEGs, 1862 and 1996 genes were successfully annotated in various databases. The analysis of RNA-seq data together with qRT-PCR validation indicated that during this transition, the genes in the oxidative phosphorylation pathway, and genes encoding P450s, serine protease inhibitor, and cuticular proteins were down-regulated, while the serine protease genes were up-regulated. Moreover, we found some 20-hydroxyecdysone (20E) biosynthesis and signaling pathway genes had a higher expression in the WS, while the genes responsible for juvenile hormone $(\mathrm{JH})$ synthesis, degradation, signaling and transporter pathways were down-regulated, suggesting these genes might be involved in the process of larval pupariation in B. dorsalis. For the chitinolytic enzymes, the genes encoding chitinases (chitinase 2, chitinase 5, chitinase 8, and chitinase 10) and chitin deacetylase might play the crucial role in the degradation of insect chitin with their expressions significantly increased during the transition. Here, we also found that chitin synthase $1 \mathrm{~A}$ might be involved in the chitin synthesis of cuticles during the metamorphosis in $B$. dorsalis.
\end{abstract}

Conclusions: Significant changes at transcriptional level were identified during the larval pupariation of $B$. dorsalis. Importantly, we also obtained a vast quantity of RNA-seq data and identified metamorphosis associated genes, which would all help us to better understand the molecular mechanism of metamorphosis process in B. dorsalis.

Keywords: Bactrocera dorsalis, Pupariation, Metamorphosis, RNA-Seq, Gene expression

\footnotetext{
* Correspondence: guy.smagghe@ugent.be; wangjinjun@swu.edu.cn

${ }^{\dagger}$ Er-Hu Chen, Qiu-Li Hou and Wei Dou contributed equally to this work.

${ }^{1}$ Key Laboratory of Entomology and Pest Control Engineering, College of

Plant Protection, Southwest University, Chongqing 400715, China

Full list of author information is available at the end of the article
}

(c) The Author(s). 2018 Open Access This article is distributed under the terms of the Creative Commons Attribution 4.0 International License (http://creativecommons.org/licenses/by/4.0/), which permits unrestricted use, distribution, and reproduction in any medium, provided you give appropriate credit to the original author(s) and the source, provide a link to the Creative Commons license, and indicate if changes were made. The Creative Commons Public Domain Dedication waiver (http://creativecommons.org/publicdomain/zero/1.0/) applies to the data made available in this article, unless otherwise stated. 


\section{Background}

The oriental fruit fly, Bactrocera dorsalis (Hendel), is a highly invasive species which has been found in India, East Asia and the Pacific region. This insect can cause significant economic losses to many commercially important tropical and subtropical crops, especially fruits, including citrus, banana, carambola, and mango [1, 2]. Because of being highly polyphagous and invasive, $B$. dorsalis has been considered as one of the most notorious pests of agricultural fruit around the world. To prevent its expansion to new host plants and geographic areas, many countries have imposed strict quarantine restrictions against $B$. dorsalis [3].

As a dipteran insect, $B$. dorsalis is a typical holometabolous insect which needs to go through larval-pupal metamorphosis to molt into adults. The metamorphosis of flies is a complex process accompanied with drastic morphological and physiological changes. Larval pupariation is the critical process during the larval-pupal development, in which the soft cuticle of a wandering larva is transformed into a hard puparium, this process involves immobilization, cuticular shrinkage, anterior retraction, longitudinal body contraction, and tanning of the cuticle $[4,5]$. It has also been reported that most of the larval tissues (integument, midgut, and fat body) must undergo a series of developmental events involving programmed cell death, and cell proliferation and differentiation to remodel structures of insects [6].

Juvenile hormone $(\mathrm{JH})$ is an important hormone in insects, which coordinates with 20-hydroxyecdysone (20E) to regulate growth and development [6]. While both of these hormones are present at larval stages, they have opposing roles in the development. For example, $\mathrm{JH}$ maintains insects at larval stage, in contrast, 20E induces metamorphosis at the end of the last larval stage when $\mathrm{JH}$ titers decrease $[7,8]$. The regulation of metamorphosis by hormones has been widely studied in many insects including Drosophila melanogaster [9], Bombyx mori [10], and Tribolium castaneum [11]. These results indeed show that the regulation of the $\mathrm{JH}$ titer in the hemolymph plays an important role in precisely mediating the process of larval-pupal metamorphosis in insects [12].

In holometabolous insects, the larval cuticle is degraded and a new cuticle of the pupal counterpart is secreted by the underlying epidermal cells during the larval-pupal metamorphosis. As chitin is the crucial component of the cuticle, the insect metamorphosis is largely dependent on chitin degradation and synthesis, which is strictly coordinated and occurs almost simultaneously within the process of molting and metamorphosis [13]. The chitin biosynthesis pathway is catalyzed by a series of enzymes, which starts with trehalose, then hexokinase, glucose-6-phosphate isomerase (G-6-P-I), glutamine-fructose-6-phosphate aminotransferase (G-F-6-P-A), glucosamine-6-phosphate
N-acetyltransferase (G-6-P-A), phosphoacetylglucosamine mutase (PM), UDP-N-acetylglucosamine pyrophosphorylase (UDP-N-A-P), and chitin synthase [14]. For the chitin catabolic pathway, chitinase, $\beta-\mathrm{N}$-acetylglucosaminidase, and chitin deacetylase are the three main enzymes that are involved in chitin degradation, which hydrolyze chitin into oligosaccharides, and further degrade these oligomers to monomers [15].

In previous studies, the gene expression patterns during metamorphosis process have been reported in many insects, including B. mori, Manduca sexta, and Spodoptera litura [16-18], however, little is known about the molecular mechanism of metamorphosis in B. dorsalis. In this paper, we elucidate the larval pupariation process by analyzing the transcriptome of $B$. dorsalis using RNA-seq at three developmental stages including the wandering stage (WS), late wandering stage (LWS, the body contraction just before pupariation), and white puparium stage (WPS). The use of RNA-seq is already well established for the analysis of gene expression in $B$. dorsalis $[3,19,20]$, and the availability of a well annotated genome of B. dorsalis (NCBI Assembly: ASM78921v2), allowed us to identify the genes differentially expressed during the different developmental stages. The results obtained through the RNA-seq analysis were further confirmed by real-time quantitative PCR. For an in-depth analysis, we focused on the genes related to oxidative phosphorylation, drug metabolism-cytochrome P450, amino acids metabolism, 20E, JH, and chitin synthesis and degradation.

\section{Methods}

\section{Insect and RNA isolation}

The stock colony of $B$. dorsalis was collected and reared according to the method described in our previously study [21]. Briefly, B. dorsalis was reared at $27 \pm 1{ }^{\circ} \mathrm{C}$ and $70 \pm 5 \%$ relative humidity with a photoperiod regime of 14:10 h light/darkness. The larvae were reared on an artificial diet containing corn, yeast powder, wheat flour as well as sucrose. A plastic basin was provided for pupation of the 3rd instar larvae. Three developmental stages, including the wandering stages (WS), late wandering stage (LWS), and white puparium stage (WPS), were collected (Fig. 1a). The 3rd-instar larvae that just jump into sand were referred as WS, the LWS was identified as the body contraction just before pupariation, and the insects that had just completed the formation of white puparium were defined as the WPS. Three replicates were performed for each stage (WS, LWS, and WPS) and each replicate contained eight flies. Total RNA was extracted by the RNeasy plus Micro Kit (Qiagen $\mathrm{GmbH}$, Hilden, Germany) following the manufacturer's instructions. In brief, the absorbance at $260 \mathrm{~nm}$ was used to quantify the RNA by a NanoVue UVVis spectrophotometer (GE Healthcare Bio-Science, Uppsala, Sweden). The absorbance ratio of OD260/280 and OD260/ 


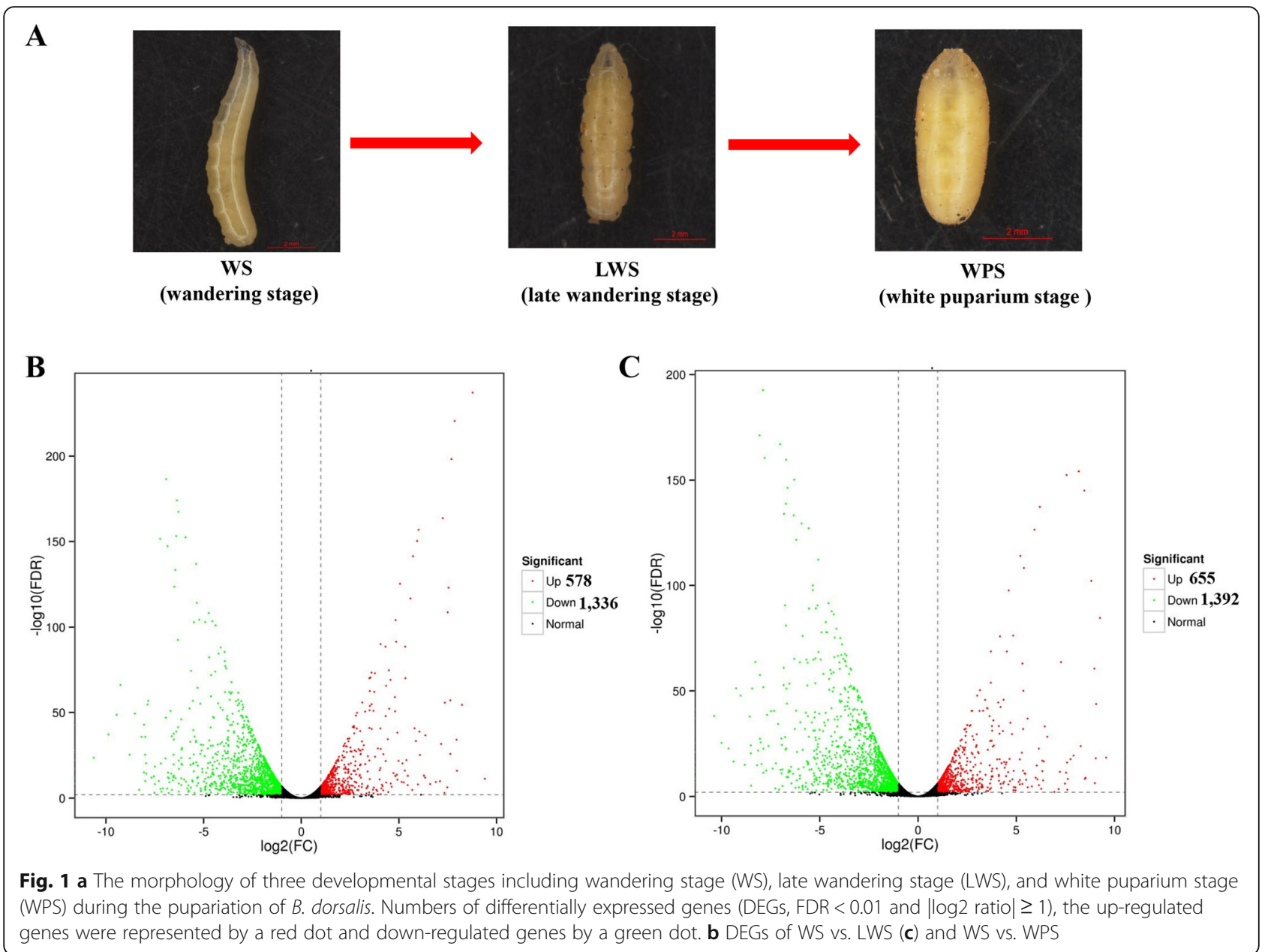

230 were used to test the purity of all RNA samples. The integrity of RNA was assessed by $1 \%$ agarose gel electrophoresis.

\section{cDNA library construction and RNA-seq sequencing}

A total amount of $1 \mu \mathrm{g}$ RNA per sample was used as input material for the RNA sample preparations. Sequencing libraries were generated using NEBNext UltraTM RNA Library Prep Kit for Illumina (NEB, Ipswich, MA, USA) following manufacturer's recommendations and index codes were added to attribute sequences to each sample.

Briefly, the poly- $\mathrm{T}$ oligo-attached magnetic beads were used for the purification of total RNA. Fragmentation was applied by using divalent cations under elevated temperature in NEBNext first strand synthesis reaction buffer $(5 \mathrm{X})$. The random hexamer primer and M-MuLV reverse transcriptase were used to synthesize the first strand cDNA. The second strand cDNA synthesis was subsequently performed using DNA Polymerase I and RNase $\mathrm{H}$. Then, the remaining overhangs were translated into blunt ends by using exonuclease/polymerase activities. The NEBNext adaptor with hairpin loop structure was ligated for further hybridization after adenylation of the 3' ends of the DNA fragments. The purification of the library fragments was performed using the AMPure XP system (Beckman Coulter, Beverly, MA, USA) to select cDNA fragments of preferentially $240 \mathrm{bp}$ in length. Afterwards, $3 \mu \mathrm{l}$ USER Enzyme (NEB) was used with size-selected, adaptor-ligated cDNA at $37{ }^{\circ} \mathrm{C}$ for 15 min followed by 5 min at $95{ }^{\circ} \mathrm{C}$ before PCR. The PCR was conducted with fusion high-fidelity DNA polymerase, universal PCR primers and index (X) Primer. Finally, PCR products were purified (AMPure XP system) and the Agilent Bioanalyzer 2100 system (Santa Clara, CA, USA) was used to evaluate library quality.

The clustering of the index-coded samples was performed on a cBot cluster generation system using the TruSeq PE Cluster Kit v4-cBot-HS (Illumina) according to the manufacturer's instructions. After cluster generation, the library preparations were sequenced on an Illumina HiSeq Xten platform and paired-end reads were generated.

\section{Analysis of RNA-seq data}

Raw data (raw reads) of fastq format were firstly processed through in-house PERL scripts. In this step, clean 
data (clean reads) were obtained by removing reads containing adapters, reads containing poly- $\mathrm{N}$ and low quality reads from raw data. At the same time, Q20, Q30, GC-content, and sequence duplication level of the clean data were calculated. These clean reads were then mapped to the reference genome sequence. Only reads with a perfect match or one mismatch were further analyzed and annotated based on the reference genome. Tophat 2 tools soft were used to map with the reference genome [22]. The gene expression levels were further quantified by FPKM (Fragment Per Kilobase of exon model per million mapped reads) method [23]. To confirm the pairs of RNA-seq results are faithful replicates, the FPKM between the biological replications was analyzed by Pearson correlation [24]. The formula is shown as follow:

$$
\text { FPKM }=\frac{\text { cDNA Fragments }}{\text { Mapped Fragments (Millions) } \times \text { Transcript Length }(\mathrm{kb})}
$$

In this study, differential expression analysis of two developmental stages was performed using the DESeq method. DESeq provides statistical routines for determining differential expression in digital gene expression data using a model based on the negative binomial distribution [25]. The resulting $P$ values were adjusted using the Benjamini and Hochberg's approach for controlling the false discovery rate (FDR). Those genes that had a two fold difference (the absolute value of $\log 2$ ratio $\geq 1$ ) between two stages and an FDR less than 0.01 were identified as differentially expressed genes (DEGs). Gene function was annotated based on the following databases: NCBI non-redundant protein sequences $(\mathrm{Nr})$, Protein family (Pfam), Clusters of Orthologous Groups of proteins (COG), a manually annotated and reviewed protein sequence database (Swiss-Prot), evolutionary genealogy of genes: Non-supervised Orthologous Groups (eggNOG), Kyoto Encyclopedia of Genes and Genomes (KEGG Ortholog database), and Gene Ontology (GO).

$\mathrm{GO}$ analysis of DEGs was constructed by the Blast2GO method [26]. Namely, the DEGs were mapped into GO terms in the database (www.geneontology.org) and calculated the gene numbers for each term. Then, significantly enriched terms were identified based on 'GO::TermFinder' (https://www.yeastgenome.org/goTermFinder). For the COG annotation, the DEGs between two developmental stages (WS vs. LWS, and WS vs. WPS) were translated into amino acid sequences using the virtual ribosome package. We also used the batch web $\mathrm{CD}$ search tool (http:// https://www.ncbi.nlm.nih.gov/Structure/bwrpsb/ bwrpsb.cgi) to assign COG groups. KEGG is a database resource for understanding high-level functions and utilities of the biological system, such as the cell, the organism and the ecosystem, and for molecular-level information, especially large-scale molecular datasets generated by genome sequencing and other high-throughput experimental technologies (http://www.genome.jp/kegg/) [27]. We used KOBAS software to test the statistical enrichment of differential expression genes in KEGG pathways [28]. Significant enrichments of the transcripts were tested by calculating the $p$-value from a Bonferroni correction at the background level of all GO, COG and KEGG classifications and we take the corrected $p$-value $<0.05$ as a threshold.

\section{Quantitative real-time polymerase chain reaction (qRT-PCR)} In this study, qRT-PCR was carried out to validate the expression of the target genes. Total RNA was extracted from the three developmental stages of WS, LWS, and WPS using TRIzol reagent. As described above, the total RNA from each stage was used for the first strand cDNA synthesis by using reverse transcriptase (Takara) and oligo $d(T)$ primer (Takara, Dalian, China). Then, Novostar-SYBR Supermix (Novoprotein, Shanghai, China) was used for qRT-PCR with a $10 \mu \mathrm{l}$ reaction system. This system consisted of $0.5 \mu \mathrm{l}$ of cDNA samples (approximately $200 \mathrm{ng} / \mathrm{mL}$ ), $0.3 \mu \mathrm{l}$ of each primer $(10 \mathrm{mM}), 5 \mu \mathrm{l}$ of Supermix, and $3.9 \mu \mathrm{l}$ of nuclease-free water. The qRT-PCR was performed with an initial 2 min denaturation at $95{ }^{\circ} \mathrm{C}$, then 40 cycles at $95{ }^{\circ} \mathrm{C}$ for $15 \mathrm{~s}$ and at $60^{\circ} \mathrm{C}$ for $30 \mathrm{~s}$ in a Mx3000P thermal cycler (Stratagene, La Jolla, CA, USA). To ensure product specificity, the melting curve was analyzed for all reactions from $60{ }^{\circ} \mathrm{C}$ to $95{ }^{\circ} \mathrm{C}$ at the end of PCR reactions. The primers used in this study were listed in Additional file 1: (Table S1). The $\alpha$-tubulin (GenBank accession number: GU269902) has the excellent stability for temporal and spatial distribution of $B$. dorsalis, and was therefore used as an internal reference gene $[29,30]$. Based on normalization with the reference gene, the $2^{-\triangle \Delta C T}$ method was used to determine the expression changes during the larval pupariation [31]. All experiments were performed in three biological replicates, the data was analyzed by one-way analysis of variance (ANOVA), and the means were separated by a least significant difference $(\mathrm{LSD})$ test $(P<0.05)$ via SPSS 16.0 software (SPSS Inc., Chicago, IL, USA).

\section{Results}

\section{Overview of the RNA-seq data}

To investigate the expression changes of genes during the larval pupariation process, we performed RNA-Seq analysis on three developmental stages (WS, LWS, and WPS) of B. dorsalis with its genome as a reference. The samples from three stages were sequenced in triplicate and a total of $74.62 \mathrm{~Gb}$ clean data was obtained. After the strict quality control, more than $99.35 \%$ of the clean data was retained. Between 70.09 and $74.44 \%$ of the reads could be mapped onto the $B$. dorsalis genome. The sequencing data has an average GC content of $41.54 \%$ and a Q30 of 91.61\% (Additional file 2: Table S2). 


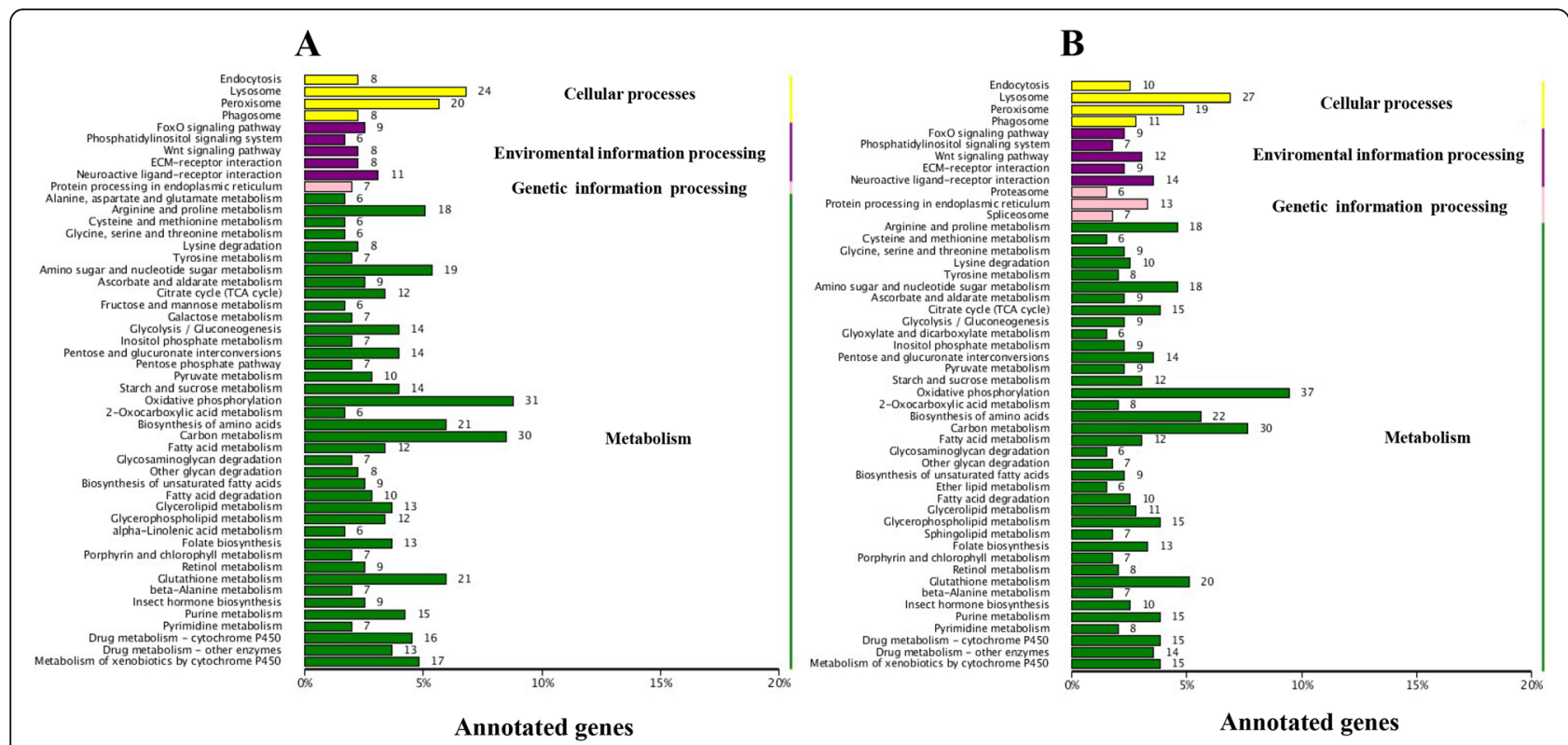

Fig. 2 KEGG pathway classification for differentially expressed genes (DEGs) during the pupariation of B. dorsalis. a KEGG pathway classification for DEGs between wandering stage (WS) and late wandering satge. b KEGG classification of DEGs in WS and white puparium stage (WPS)

\section{Analysis of differentially expressed genes (DEGs)}

FPKM was calculated and standardized for the analysis of gene expression, and similar patterns of FPKM density were obtained for each sample of $B$. dorsalis, suggesting high reproducibility of the transcriptome analysis of each developmental stage (Additional file 3: Figure. S1). In total 11,721 genes were identified from the 9 samples and their gene expression profiles are shown in Additional file 4: (Table S3). There were 11,301 genes that could be annotated in $B$. dorsalis genome, this is $83.76 \%$ of the total predicted genes. We also found 420 genes that could not be annotated in $B$. dorsalis genome, and were viewed as the novel genes.

In this study, we found significant gene expression changes (two fold difference between two stages with an FDR less than 0.01) accompanying pupariation in B. dorsalis (Fig. 1b and c). From WS to LWS (WS vs. LWS), 1914 genes were identified as differentially expressed, among which 578 were up-regulated and 1336 were down-regulated (Fig. 1b). From WS to WPS (WS vs. WPS), 2047 genes were identified as differentially expressed, of which 655 were up-regulated and 1392 were down-regulated (Fig. 1c). However, from LWS to WPS (LWS vs. WPS), only 160 genes were detected differentially expressed with 65 were up-regulated and 95 were down-regulated (Additional file 5: Figure. S2), suggesting less changes at molecular levels between these two stages. Therefore, we focused on the analysis of DEGs between WS vs. LWS and WS vs. WPS.

\section{GO, COG and KEGG analysis of differentially expressed} genes (DEGs)

To explore the potential function of the DEGs during pupariation, seven databases were used, including COG, GO, KEGG, Nr, Pfam, Swiss-Prot, and eggNOG (Additional file 6: Figure. S3). As there was the large overlap of DEGs between WS vs. LWS and WS vs. WPS, similar annotations were identified for each database, and totally 1862 (Additional file 7: Table S4) and 1996 (Additional file 8: Table S5) genes were successfully annotated, respectively. For the GO classification, the DEGs of two comparisons (WS vs. LWS and WS vs. WPS) were both divided into three ontologies: biological process, cellular component, and molecular function, including 49 annotations for each comparison (Additional file 9: Figure. S4 and Additional file 10: Figure. S5). Furthermore, a COG functional classification was performed to compare the enriched functional categories of the DEGs between WS and LWS or WS and WPS using the rpstBlastn program [32]. When mapping all the DEGs into the COG database, a total of 624 (WS vs. LWS) and 667 (WS vs. WPS) genes were annotated, which had a similar COG classification (Additional file 11: Figure. S6). The two DEGs profiles were each annotated into 22 COG categories, and the largest group in the cluster was 'general function prediction only', followed by 'Amino acid transport and metabolism, 'Carbohydrate transport and metabolism,' 'Inorganic ion transport and metabolism,' 'Secondary metabolites biosynthesis, transport and catabolism, 'Lipid transport and metabolism,' 'Signal transduction mechanisms', and 'Energy production and conversion'. 
Here, the DEGs of WS vs. LWS and WS vs. WPS were each mapped to the KEGG pathways, respectively, a total of 658 and 722 genes were classified into 50 pathways (Fig. 2). Similar to the COG annotation, the metabolism pathways is also the largest classification in the KEGG analysis, which contained 'oxidative phosphorylation,' 'carbon metabolism,' 'biosynthesis of amino acids', 'glutathione metabolism', and 'amino sugar and nucleotide sugar metabolism'. Furthermore, two pathways of 'drug metabolism-cytochrome $\mathrm{P} 450$ ' and 'metabolism of xenobiotics by cytochrome P450' were involved in metabolism classification as well (Fig. 2). For the cellular processes and environmental information processing, the 'endocytosis', 'lysosome,' 'peroxisome' and 'phagosome', and the 'FoxO signaling,' 'phosphatidylinositol signaling system,' 'wnt signaling,' 'ECM-receptor interaction' and 'neuroactive ligand-receptor interaction' pathways were identified respectively. As for the genetic information processing, only the 'protein processing in endoplasmic reticulum' pathway was annotated for DEGs (WS vs. LWS), and three pathways including 'proteasome,' 'protein processing in endoplasmic reticulum' and 'spliceosome' were annotated for DEGs (WS vs. WPS). Importantly, the KEGG pathway enrichment analysis showed that the 'amino sugar and nucleotide sugar metabolism' pathway was significantly enriched for the up-regulated genes (WS vs. LWS and WS vs. WPS) (Fig. 3a and c), and the 'oxidative phosphorylation' and 'insect hormone biosynthesis' pathways were significant enriched for the down-regulated genes (Fig. 3b and d). Generally speaking, the GO, COG and KEGG analysis give us an overview of function of DEGs, and the pathways described above might play an important role in the process of pupariation in $B$. dorsalis.

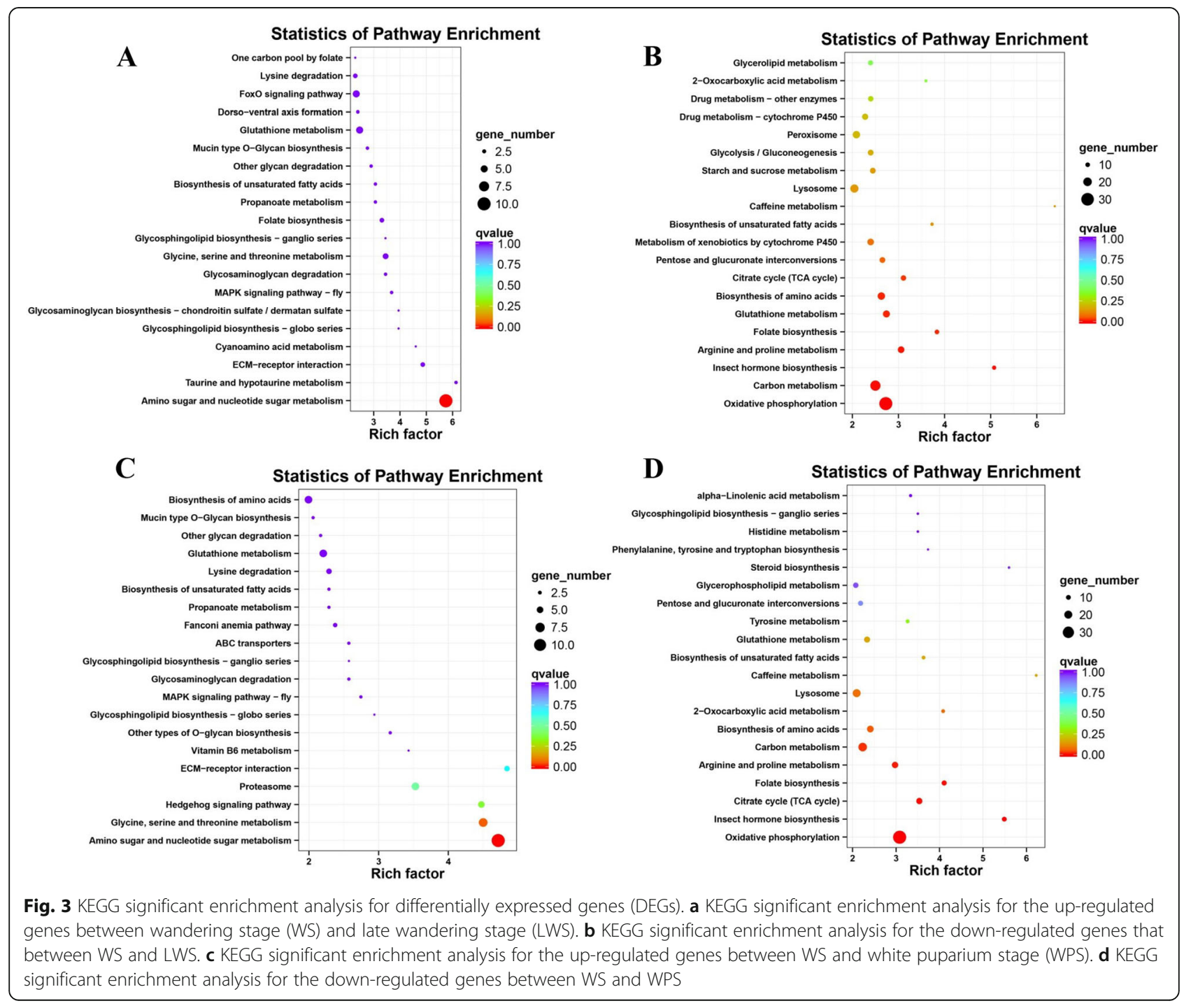


Changes of the gene expressions in the oxidative phosphorylation pathway

The genes in the oxidative phosphorylation pathway were significantly down-regulated during the pupariation of $B$. dorsalis, specifically the genes encoding NADH dehydrogenase, succinate dehydrogenase, cytochrome c reductase, cytochrome c oxidase, and ATPase (Fig. 4).

\section{Changes in expression level of P450 genes}

A large number of P450 genes were significantly down-regulated during the larval pupariation in $B$. dorsalis, especially for CYP4 and CYP6 members (Table 1). Interestingly, we also found three CYP3 genes that were significantly up-regulated (Table 1).

Changes of the gene expressions in the 20-hydroxyecdysone (20E) and juvenile hormone $(\mathrm{JH})$ pathways

Four genes encoding CYP302a1, CYP306a1, CYP307a1 and CYP314a1, involved in 20E biosynthesis, and three
20E signaling pathway genes encoding E74, E75, and FTZ-F1 had the highest expressions in the wandering stage (Fig. 5 and Additional file 12: Table S6). The JH synthesis pathway contains a series of enzymatic reactions and in total 10 genes could be detected during the larval metamorphosis in $B$. dorsalis (Table 2). Especially the expression of the three genes encoding farnesol dehydrogenase (FD), farnesoic acid O-methyl transferase (FAOMT) and juvenile hormone acid O-methyltransferase (JHAOM) was significantly decreased in the process of pupariation. Interestingly, we found three key enzymes $(\mathrm{JH}$ epoxide hydrolase, $\mathrm{JH}$ diol kinase, and $\mathrm{JH}$ esterase) involved in the degradation of $\mathrm{JH}$ that were significantly down-regulated in $B$. dorsalis (Table 2). As the key genes in the $\mathrm{JH}$ signal transduction pathway, the gene expressions of methoprene-tolerant (Met), Krüppel homolog 1 (Kr-h1) and steroid receptor coactivator (SRC) were significantly down-regulated during the pupariation in B. dorsalis as well (Table 2). Moreover,

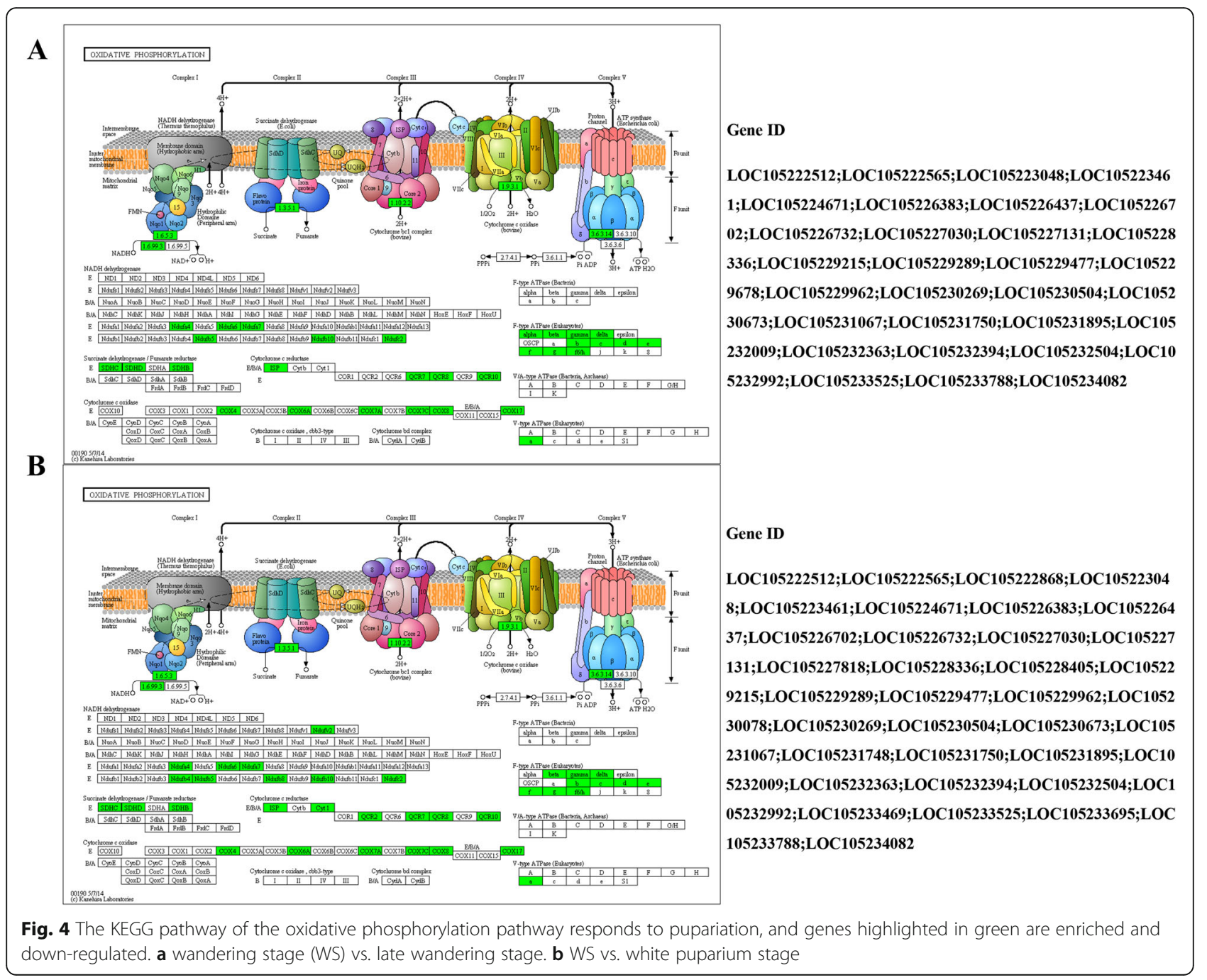


Table 1 Expression level of the P450 genes during the pupariation in Bactrocera dorsalis

\begin{tabular}{|c|c|c|c|c|c|c|}
\hline Gene ID & WS-FPKM & LWS-FPKM & WPS-FPKM & WS vs LWS & WS vs WPS & Blast nr \\
\hline \multicolumn{7}{|c|}{ Down-regulated genes } \\
\hline LOC105230198 & 6.14 & 0.11 & 0.25 & -6.10 & -4.67 & cytochrome P450 6a13 \\
\hline LOC105233810 & 1577.65 & 48.39 & 76.59 & -5.02 & -4.37 & cytochrome P450 6 g2 \\
\hline LOC109579301 & 92.94 & 4.84 & 3.95 & -4.54 & -4.27 & cytochrome P450 6 g1-like \\
\hline LOC105222599 & 405.68 & 20.38 & 16.49 & -4.39 & -4.68 & cytochrome P450 6 g1-like \\
\hline LOC105228198 & 3.01 & 0.20 & 0.11 & -4.14 & -4.81 & cytochrome P450 6a9-like \\
\hline LOC105233910 & 59.36 & 3.82 & 18.24 & -3.95 & -1.70 & cytochrome P450 4c3 \\
\hline LOC105230949 & 152.70 & 10.16 & 7.75 & -3.91 & -4.28 & cytochrome P450 6a14 \\
\hline LOC105225363 & 190.01 & 13.39 & 15.48 & -3.85 & -3.64 & cytochrome P450 9b2-like \\
\hline LOC105230203 & 61.83 & 5.34 & 3.85 & -3.53 & -4.00 & cytochrome P450 6a9-like \\
\hline LOC105230201 & 22.22 & 2.09 & 0.98 & -3.46 & -4.58 & cytochrome P450 6a21 \\
\hline LOC105231082 & 81.51 & 8.77 & 5.23 & -3.24 & -4.01 & cytochrome P450 12e1, mitochondrial \\
\hline LOC105232227 & 8.40 & 0.92 & 1.61 & -3.23 & -2.36 & cytochrome P450 311a1 \\
\hline LOC105231049 & 1370.38 & 159.16 & 56.06 & -3.17 & -4.67 & cytochrome P450 304a1 \\
\hline LOC105228053 & 16.88 & 1.96 & 2.48 & -3.15 & -2.78 & cytochrome P450 4ad1 \\
\hline LOC105231113 & 205.39 & 23.07 & 9.89 & -3.08 & -4.06 & cytochrome P450 12e1 \\
\hline LOC105224924 & 39.70 & 5.56 & 11.36 & -2.87 & -1.82 & cytochrome P450 18a1 \\
\hline LOC105231454 & 27.69 & 5.56 & 10.06 & -2.36 & -1.44 & cytochrome P450 4d8 \\
\hline LOC105230935 & 48.89 & 10.80 & 13.69 & -2.19 & -1.85 & cytochrome P450 6d5 \\
\hline LOC105230199 & 12.80 & 2.85 & 3.38 & -2.16 & -1.91 & cytochrome P450 317a1 \\
\hline LOC105230713 & 7.86 & 1.78 & 1.91 & -2.15 & -2.03 & cytochrome P450 301a1, mitochondrial \\
\hline LOC105226210 & 53.21 & 13.39 & 10.29 & -2.02 & -2.39 & cytochrome P450 12c1, mitochondrial \\
\hline LOC109580018 & 17.03 & 4.82 & 2.61 & -2.01 & -3.05 & cytochrome P450 313a4 \\
\hline LOC105234039 & 254.72 & 70.14 & 60.75 & -1.88 & -2.16 & cytochrome P450 309a1 \\
\hline LOC105227474 & 221.04 & 60.58 & 48.65 & -1.84 & -2.15 & cytochrome P450 6a2-like \\
\hline LOC105228051 & 79.19 & 23.67 & 23.14 & -1.76 & -1.80 & cytochrome P450 4e2-like \\
\hline LOC105225824 & 50.18 & 10.47 & 12.11 & -1.73 & -1.66 & cytochrome P450 9f2 \\
\hline LOC105226035 & 170.22 & 52.36 & 53.18 & -1.71 & -1.70 & cytochrome P450 6d4 \\
\hline LOC105225364 & 262.08 & 82.38 & 56.90 & -1.68 & -2.24 & cytochrome P450 9b2-like \\
\hline LOC105225361 & 30.28 & 9.72 & 15.56 & -1.68 & -1.01 & cytochrome P450 9 h1 \\
\hline LOC105230202 & 30.33 & 9.71 & 10.32 & -1.66 & -1.54 & cytochrome P450 6a21 \\
\hline LOC105229174 & 37.97 & 12.68 & 8.74 & -1.64 & -2.18 & cytochrome P450 4p1-like \\
\hline LOC105227668 & 27.04 & 8.80 & 6.78 & -1.62 & -2.00 & cytochrome P450 4aa1 \\
\hline LOC105224875 & 10.38 & 4.78 & 4.89 & -1.11 & -1.05 & cytochrome P450 302a1, mitochondrial \\
\hline \multicolumn{7}{|l|}{ Up-regulated genes } \\
\hline LOC105226220 & 1.37 & 13.26 & 61.18 & 3.28 & 5.50 & cytochrome P450 12b2, mitochondrial \\
\hline LOC105227996 & 5.00 & 29.60 & 30.28 & 2.81 & 2.77 & cytochrome P450 313a4 \\
\hline LOC105227639 & 77.80 & 233.28 & 440.26 & 1.57 & 2.50 & cytochrome P450 12a4, mitochondrial \\
\hline LOC105226219 & 45.76 & 134.86 & 207.65 & 1.56 & 2.18 & cytochrome P450 12b1, mitochondrial-like \\
\hline LOC105230977 & 6.85 & 13.84 & 13.31 & 1.22 & 1.14 & cytochrome P450 313a4 \\
\hline LOC105231048 & 541.23 & 1207.42 & 1104.91 & 1.14 & 1.03 & cytochrome P450 304a1 \\
\hline
\end{tabular}




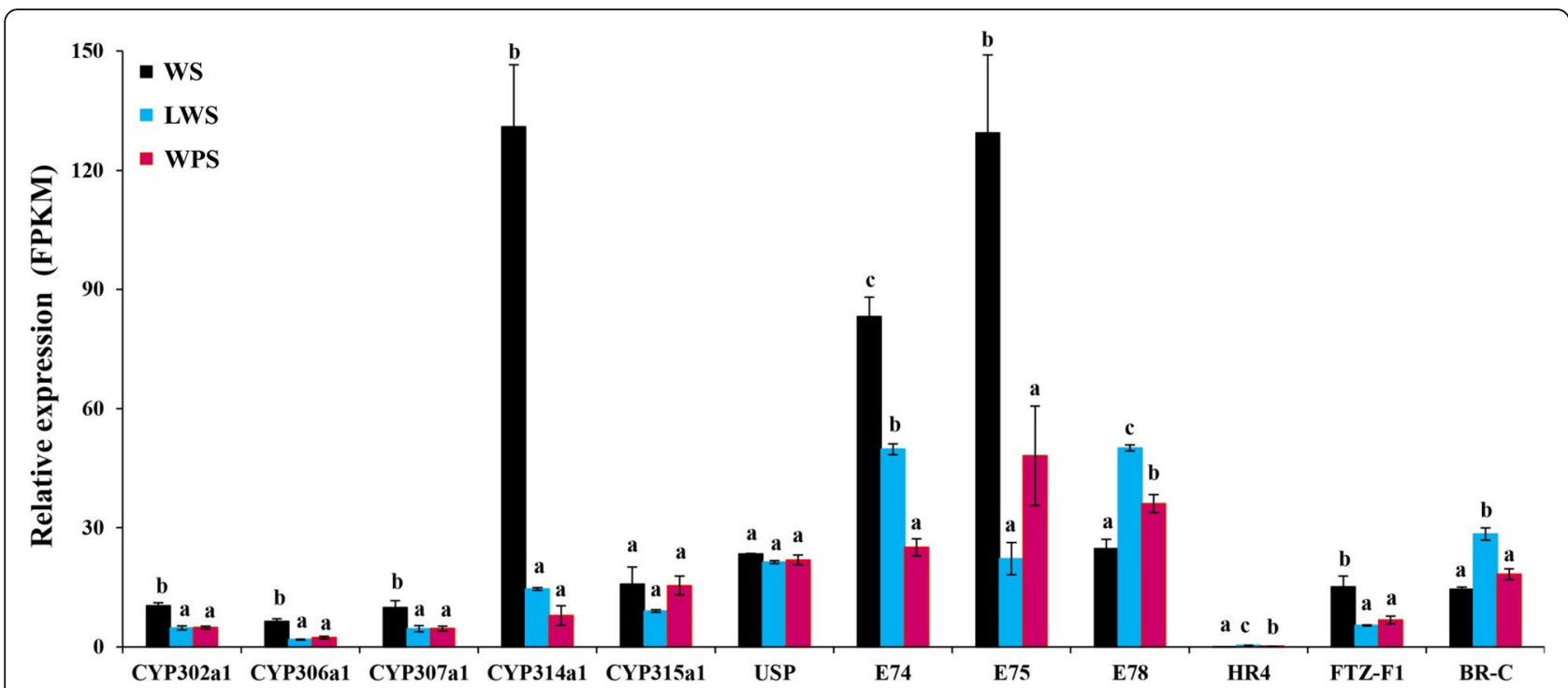

Fig. 5 The expression patterns (FPKM value) of five 20E biosynthesis and seven signaling pathway genes during the pupariation in Bactrocera dorsalis. Three replications were conducted, and the data are presented as mean \pm SE. Significant differences among the three treatments were analyzed by one-way analysis. Bars with different letters above them differ significantly at $P<0.05$

the $\mathrm{JH}$ transport pathway was significantly blocked, with the transcript levels of the JHBP genes significantly decreased during the larval metamorphosis in B. dorsalis (Table 2).

\section{Change in the gene expressions in the chitin degradation and synthesis pathways}

Three serine protease genes were found to be up-regulated and five genes of serine protein inhibitors were down-regulated (Table 3). Interestingly, expression levels of a large number of cuticular protein genes were found to be significantly decreased as well (Table 4). Here, the results of RNA-seq (Table 5) and qRT-PCR (Fig. 6a) showed that the gene expression of four chitinase (chitinase 2 , chitinase 5 , chitinase 8 , and chitinase 10) and chitin deacetylase was significantly increased upon pupariation, indicating their important role in the larval metamorphosis. However, the gene expression patterns of chitinase 3 and $\beta-\mathrm{N}$-acetylglucosaminidase were different from the above five enzymes, and for these two, mRNA levels were the highest in larval stage, which implied their possible role in the degradation of larval cuticle (Table 5 and Fig. 6a). The expression of genes that are related with the chitin biosynthesis pathway are shown in Table 6 and Fig. 6b, respectively. Based on the qRT-PCR result, we found that most of the genes encoding trehalase, hexokinase, G-F-6-P-A, G-6-P-A, PM, and chitin synthase $1 \mathrm{~A}$ were significantly up-regulated during the pupariation, except for the genes encoding G-6-P-I, UDP-N-A-P, chitin synthase 1B, and chitin synthase 2 (Fig. 6b).

\section{Discussion}

In our previous study, the RNA-seq libraries have been constructed without a reference genome to investigate the gene expression patterns at different developmental stages from eggs to adults (eggs, larvae, pupae, and adults) [3]. Because the lack of an annotated $B$. dorsalis genome, many DEGs were found to encode proteins with unknown function among the different developmental stages, suggesting insufficient knowledge of the molecular mechanisms underlying the development of $B$. dorsalis. Currently, the $B$. dorsalis genome is available online (NCBI Assembly: ASM78921v2) and a total of 11,301 genes were identified during larval pupariation, with a large number of genes changing during the LWS and WPS relative to the WS. Importantly, this work is the first transcriptome exploration of metamorphosis in B. dorsalis, which has provided us with this useful gene expression resource to further understand the metamorphosis development in this species.

As the pupa is a relatively stable and immobile stage, the larval stage is the critical stage to obtain energy to complete the metamorphosis [33], and the energy derived from larvae can also make a significant contribution to the reproductive output during their entire life $[34,35]$. Therefore, many previous studies have reported that energy metabolic rates declined sharply after pupariation, and are maintained at a low level until the eclosion to the adult $[36,37]$. Similarly, the present KEGG analysis also confirmed the previous study in D. melanogaster [38], that the oxidative phosphorylation pathway was significantly blocked, indicating less production of 
Table 2 Expression level of the juvenile hormone related genes during the pupariation in Bactrocera dorsalis

\begin{tabular}{|c|c|c|c|c|c|c|}
\hline Gene ID & WS-FPKM & LWS-FPKM & WPS-FPKM & WS vs LWS & WS vs WPS & Blast nr \\
\hline \multicolumn{7}{|c|}{ juvenile hormone synthesis pathway } \\
\hline LOC105226875 & 158.49 & 193.63 & 216.66 & + & + & Acetoacetyl-CoA thiolase \\
\hline LOC105227740 & 11.83 & 6.01 & 5.89 & - & $-1.03^{*}$ & 3-Hydroxy-3-methylglutaryl-CoA synthase \\
\hline LOC105226753 & 2.91 & 4.38 & 4.36 & + & + & 3-Hydroxy-3-methylglutaryl-CoA reductase \\
\hline LOC105233868 & 39.12 & 30.559 & 33.79 & - & - & Mevalonate kinase \\
\hline LOC105230627 & 20.54 & 23.40 & 23.82 & + & + & Phosphomevalonate kinase \\
\hline LOC105224988 & 14.42 & 12.11 & 11.58 & - & - & Diphosphomevalonate decarboxylase \\
\hline LOC105230652 & 24.54 & 27.08 & 26.39 & + & + & Farnesyl diphosphate synthase \\
\hline LOC105225547 & 70.82 & 27.06 & 24.24 & $-1.41^{*}$ & $-1.50^{*}$ & Farnesol dehydrogenase \\
\hline LOC105228451 & 683.52 & 151.47 & 159.65 & $-2.16^{*}$ & $-2.01^{*}$ & Farnesoic acid O-methyl transferase \\
\hline LOC105227166 & 18.26 & 3.77 & 4.05 & $-2.39^{*}$ & $-2.30^{*}$ & Juvenile hormone acid O-methyltransferase \\
\hline \multicolumn{7}{|c|}{ Juvenile hormone degradation pathway } \\
\hline LOC105230544 & 15.22 & 2.97 & 2.07 & $-2.38^{*}$ & $-2.87^{*}$ & Juvenile hormone epoxide hydrolase \\
\hline LOC105226721 & 1517.31 & 230.58 & 104.88 & $-2.78^{*}$ & $-3.94^{*}$ & Juvenile hormone diol kinase \\
\hline LOC105229968 & 0.99 & 0.15 & 0.034 & $-2.90^{*}$ & $-5.00^{*}$ & Juvenile hormone esterase \\
\hline \multicolumn{7}{|c|}{ Juvenile hormone signal transduction pathway } \\
\hline LOC 105228252 & 22.35 & 20.74 & 15.64 & - & - & Methoprene-tolerant \\
\hline LOC105232250 & 26.87 & 18.75 & 14.51 & - & - & Steroid receptor coactivator \\
\hline LOC105224804 & 23.21 & 15.81 & 14.51 & - & - & Krüppel homolog 1 \\
\hline \multicolumn{7}{|c|}{ Juvenile hormone transporter pathway } \\
\hline LOC105232271 & 423.59 & 2.15 & 2.09 & $-7.67^{*}$ & $-7.72^{*}$ & Juvenile hormone binding protein \\
\hline LOC105234182 & 1134.50 & 7.68 & 4.28 & $-7.22^{*}$ & $-8.06^{*}$ & Juvenile hormone binding protein \\
\hline LOC109579562 & 2.75 & 0.047 & 0 & $-6.86^{*}$ & * & Juvenile hormone binding protein \\
\hline LOC105232270 & 2659.47 & 38.67 & 19.20 & $-6.13^{*}$ & $-7.11^{*}$ & Juvenile hormone binding protein \\
\hline LOC105232273 & 2559.46 & 61.87 & 23.55 & $-5.37^{*}$ & $-6.76^{*}$ & Juvenile hormone binding protein \\
\hline LOC105234180 & 19.13 & 0.63 & 1.57 & $-5.13^{*}$ & $-3.70^{*}$ & Juvenile hormone binding protein \\
\hline LOC105232274 & 71.64 & 2.85 & 1.11 & $-4.67^{*}$ & $-6.01^{*}$ & Juvenile hormone binding protein \\
\hline LOC105232427 & 143.97 & 7.50 & 3.81 & $-4.28^{*}$ & $-5.24^{*}$ & Juvenile hormone binding protein \\
\hline LOC105226548 & 11.19 & 1.13 & 1.24 & $-3.40^{*}$ & $-3.22^{*}$ & Juvenile hormone binding protein \\
\hline LOC109579964 & 951.84 & 145.71 & 84.49 & $-2.75^{*}$ & $-3.50^{*}$ & Juvenile hormone binding protein \\
\hline LOC105226550 & 130.82 & 26.86 & 33.09 & $-2.29^{*}$ & $-2.01^{*}$ & Juvenile hormone binding protein \\
\hline LOC105226544 & 10.54 & 2.66 & 2.26 & $-2.04^{*}$ & $-2.31^{*}$ & Juvenile hormone binding protein \\
\hline LOC105231611 & 10.53 & 3.77 & 3.38 & $-1.51^{*}$ & $-1.71^{*}$ & Juvenile hormone binding protein \\
\hline
\end{tabular}

The gene expression level was calculated by RPKM method. " + " represents up-regulation, and "-" represents down-regulation. Asterisks (*) indicate that the gene, whose expression level had more than two folds difference between two stages and False Discovery Rate (FDR) was less than 0.01

energy in LWS and WPS, when comparing to WS in $B$. dorsalis. One of the explanations might be that the WS is a very active stage that aims to find the suitable place for pupariation, on the contrary, the LWS and WPS are both immobile stages, which certainly require less energy supply in these two stages of $B$. dorsalis. Another possible explanation is that energy stores in larvae are limited, and the energy metabolism has to be decreased, otherwise the pupa could simply run out of energy. Moreover, energy stores acquired from the larval stage were retained in the pupa, and would be present to support the newly-eclosed fly adult, as flies do not feed at the beginning of their adult lives $[39,40]$. The present result also proves the U-shaped metabolic curve, which is necessary for insect metamorphosis [41].

Interestingly, we also found the expressions of many CYP genes decreased sharply, a similar result has also been reported in S. litura, showing that CYP4 and CYP6 groups were predominantly expressed in wandering stage and had a lower expression level in prepupae or pupae stage [18]. The CYP4 and CYP6 family contains the largest number of CYP members, and the genes 
Table 3 Expression level of the serine protease and inhibitor genes during the pupariation in Bactrocera dorsalis

\begin{tabular}{lllllll}
\hline Gene ID & WS-FPKM & LWS-FPKM & WPS-FPKM & WS vs LWS & WS vs WPS & Blast nr \\
\hline Up-regulated & & & & & & \\
LOC105227168 & 0.029 & 0.48 & 1.78 & 4.20 & 6.08 & serine protease nudel \\
LOC105226969 & 2.25 & 36.35 & 51.87 & 4.01 & 4.52 & serine protease persephone-like \\
LOC105224105 & 76.39 & 192.55 & 217.22 & 1.34 & 1.51 & serine protease easter-like \\
Down-regulated & & & & & -4.35 & serine protease inhibitor \\
LOC105229202 & 78.18 & 5.39 & 4.37 & -4.02 & -3.48 & serine protease inhibitor \\
LOC105234027 & 36.95 & 4.22 & 3.32 & -3.12 & -2.49 & serine protease inhibitor \\
LOC105224656 & 41.61 & 5.48 & 7.52 & -2.97 & -2.10 & serine protease inhibitor \\
LOC105224655 & 256.05 & 68.06 & 59.24 & -1.92 & -1.26 & serine protease inhibitor \\
LOC105224653 & 60.96 & 17.71 & 25.93 & -1.86 &
\end{tabular}

The criteria applied for significance difference are $\mathrm{FDR} \leq 0.01$, and estimated absolute $\mid \log 2$ Ratio $\mid \geq 1$

from these two families are mainly involved in the metabolism of xenobiotics and endogenous toxins in D. melanogaster [42] and Anopheles funestus [43]. As the puparium offers a physical barrier to protect the immature stages from exposure to xenobiotics, the lower expressions of CYP genes are rational.
Some research reported that the CYP3 genes were involved in cuticle formation by the regulation of ecdysone in D. melanogaster. Here, the expressions of three CYP3 genes increased significantly, suggesting their possible role in cuticle formation during the pupariation in B. dorsalis.

Table 4 Expression level of the cuticular protein genes during the pupariation in Bactrocera dorsalis

\begin{tabular}{|c|c|c|c|c|c|c|}
\hline Gene ID & WS-FPKM & LWS-FPKM & WPS-FPKM & WS vs LWS & WS vs WPS & Blast nr \\
\hline \multicolumn{7}{|c|}{ Down-regulated genes } \\
\hline LOC105222609 & 13.95 & 0 & 0.16 & & -6.74 & larval cuticle protein $A 2 B$ \\
\hline LOC105231490 & 20.82 & 0 & 0.12 & & -6.82 & cuticle protein LPCP-23 \\
\hline LOC105233876 & 338.64 & 1.56 & 1.31 & -7.84 & -8.05 & cuticular proteins \\
\hline LOC105232796 & 439.91 & 5.19 & 16.49 & -6.45 & -4.68 & endocuticle structural glycoprotein SgAbd-3 \\
\hline LOC105229609 & 26.99 & 1.49 & 1.07 & -4.54 & -4.85 & Larval cuticle protein 9 \\
\hline LOC105228055 & 2595.35 & 131.71 & 44.33 & -4.38 & -5.93 & larval cuticle protein 2-like \\
\hline LOC105228057 & 33.16 & 1.90 & 1.59 & -4.26 & -4.42 & larval cuticle protein 2-like \\
\hline LOC105222608 & 137.42 & 10.35 & 10.72 & -3.81 & -3.71 & larval cuticle protein $A 2 B$ \\
\hline LOC105232742 & 26.60 & 2.40 & 0.93 & -3.64 & -5.17 & larval cuticle protein 5-like \\
\hline LOC105233885 & 97.17 & 10.27 & 7.32 & -3.59 & -3.97 & Larval cuticle protein 9 \\
\hline LOC105228103 & 207.33 & 20.15 & 31.77 & -3.52 & -2.54 & larval cuticle protein LCP-17 \\
\hline LOC105227146 & 3.35 & 0.39 & 0.28 & -3.27 & -3.70 & cuticle protein Edg-78E-like \\
\hline LOC105232751 & 343.93 & 20.57 & 6.29 & -3.09 & -4.52 & endocuticle structural glycoprotein SgAbd-5 \\
\hline LOC105222606 & 5.84 & 0.97 & 0.089 & -2.76 & -6.94 & Larval cuticle protein $\mathrm{A} 2 \mathrm{~B}$ \\
\hline LOC105228054 & 47.12 & 7.82 & 12.29 & -2.67 & -2.00 & larval cuticle protein 2-like \\
\hline LOC105223825 & 261.60 & 59.42 & 45.43 & -2.17 & -2.50 & cuticle protein 16.8 \\
\hline LOC105228110 & 802.08 & 263.54 & 270.89 & -1.68 & -1.62 & endocuticle structural protein SgAbd-6-like \\
\hline LOC105232738 & 23.53 & 11.22 & 10.14 & -1.23 & -1.36 & larval cuticle protein 5-like \\
\hline LOC105233894 & 4134.03 & 1868.15 & 1170.25 & -1.23 & -1.91 & larval cuticle protein LCP-30 \\
\hline LOC105224878 & 6.93 & 3.18 & 3.14 & -1.11 & -1.19 & Insect cuticle protein \\
\hline \multicolumn{7}{|l|}{ Up-regulated genes } \\
\hline LOC105225950 & 0.15 & 67.39 & 71.21 & 9.40 & 9.57 & Endocuticle structural glycoprotein SgAbd-9 \\
\hline LOC105223248 & 0.98 & 1.17 & 1.48 & 3.72 & 4.12 & Insect cuticle protein \\
\hline
\end{tabular}


Table 5 Expression level of the chitin degradation related genes during the pupariation in Bactrocera dorsalis

\begin{tabular}{|c|c|c|c|c|}
\hline Gene ID & WS-FPKM & LWS-FPKM & WPS-FPKM & Blast nr \\
\hline LOC105224788 & 36.89 & 33.07 & 48.72 & chitinase 2 \\
\hline LOC105229469 & 207.34 & 6.98 & 2.60 & chitinase 3 \\
\hline LOC105226345 & 14.70 & 228.71 & 586.63 & chitinase 5 \\
\hline LOC105232395 & 13.69 & 88.89 & 60.02 & chitinase 8 \\
\hline LOC105232357 & 7.59 & 58.13 & 57.74 & chitinase 10 \\
\hline LOC105225231 & 84.25 & 46.72 & 182.69 & chitin deacetylase \\
\hline LOC105227151 & 22.33 & 5.18 & 6.92 & $\beta$-N-acetylglucosaminidase \\
\hline
\end{tabular}

In insects, $20 \mathrm{E}$ and $\mathrm{JH}$ are the two primary hormones to regulate different biological processes of growth, molting, metamorphosis, and reproduction [16]. The steroid 20E is the molting hormone, which could trigger the molting process and mediate the morphogenetic processes including the metamorphosis in insects [44]. CYP302a1, CYP306a1, CYP307a1 and CYP314a1 are known as the halloween genes, and are involved in the final steps of 20E biosynthetic pathway, catalyzing the conversion of ecdysone to 20E [45]. The RNAi-mediated knockdown of specific halloween genes results in morphological defects such as precocious metamorphosis, impaired nymphs, and cuticle deformities that are accompanied with a decrease of $20 \mathrm{E}$ level [45-47]. The $20 \mathrm{E}$ signal pathway is composed of nuclear receptors, such as USP, E74, E75, E78, and FTZ-F1 that can strictly

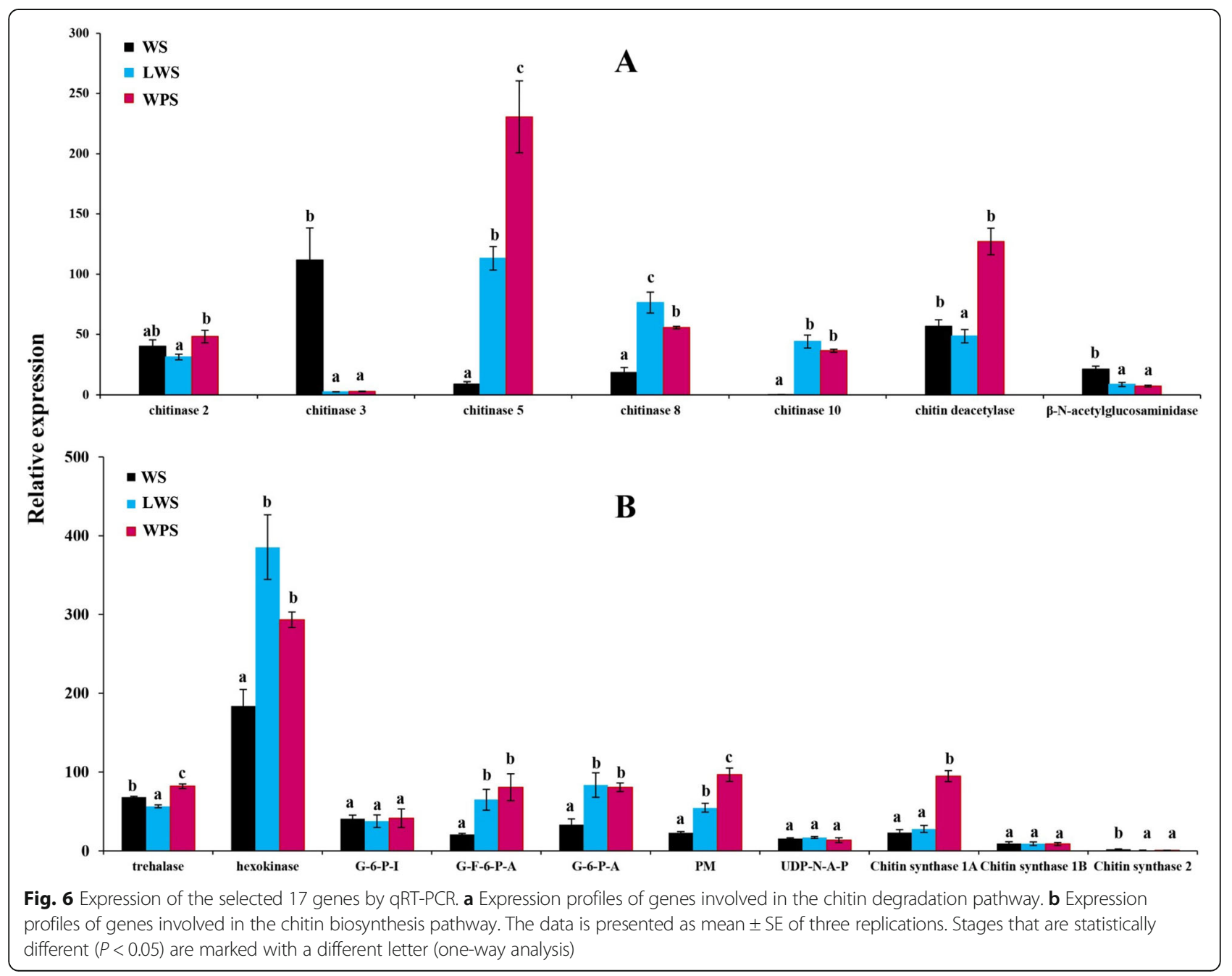


Table 6 Expression levels of the chitin synthesis related genes during pupariation in Bactrocera dorsalis

\begin{tabular}{lllll}
\hline Gene ID & WS-FPKM & LWS-FPKM & WPS-FPKM & Blast nr \\
\hline LOC105228449 & 60.60 & 48.09 & 71.89 & trehalase \\
LOC105223186 & 126.74 & 292.94 & 189.01 & hexokinase type 2 \\
LOC105228090 & 41.35 & 43.35 & 43.93 & glucose-6-phosphate isomerase \\
LOC105231918 & 26.40 & 125.20 & 134.79 & glutamine--fructose-6-phosphate aminotransferase \\
LOC105223703 & 94.87 & 138.05 & 142.47 & glucosamine-6-phosphate N-acetyltransferase \\
LOC105225295 & 35.89 & 88.83 & 103.41 & phosphoacetylglucosamine mutase \\
LOC105233954 & 60.62 & 53.57 & 52.58 & UDP-N-acetylhexosamine pyrophosphorylase \\
LOC105224260 & 48.31 & 59.35 & 196.76 & Chitin synthase1A \\
LOC105226562 & 3.28 & 2.86 & 3.96 & Chitin synthase 1B \\
LOC105224261 & 2.75 & 0.99 & 0.59 & Chitin synthase 2 \\
\hline
\end{tabular}

control insect metamorphosis [18]. In the current study, we found a higher expression of $20 \mathrm{E}$ biosynthesis and signaling pathway genes in WS, as this stage is the proecdysis of $B$. dorsalis larvae, we believe that the higher expression of these genes is crucial to initiate molting process during the pupariation in $B$. dorsalis.

$\mathrm{JH}$ is a unique insect hormone, which is synthesized and released from a small group of glands that is located posterior to the brain, called corpora allata (CA) [48]. It has been reported that $\mathrm{JH}$ is involved in the regulation of insect molting and metamorphosis by modulating ecdysone action. Previous studies have revealed that changes of $\mathrm{JH}$ titers are primarily controlled through the $\mathrm{JH}$ synthesis and degradation pathways by the action of specific enzymes [49]. In this study, we found that the $\mathrm{JH}$ related genes including synthesis, degradation, signal transduction, and transporter pathways were all down-regulated in the process of metamorphosis, indicating their critical role in the pupariation of $B$. dorsalis. FD is a rate limiting enzyme for JH biosynthesis, which is involved in the oxidation of farnesol to farnesoic acid, suggesting its importance in the regulation of $\mathrm{JH}$ titer [50]. Methyl farnesoate is the immediate precursor of $\mathrm{JH}$, and the FAOMT enzyme could catalyze methylation of farnesoic acid into methyl farnesoate, which is the penultimate step of insect $\mathrm{JH}$ biosynthesis and thus an important regulator in insect development [51]. As the final step of $\mathrm{JH}$ biosynthesis, JHAOM is specifically expressed in the $\mathrm{CA}$ and its temporal expression strongly correlates with the $\mathrm{JH}$ biosynthetic activity, indicating this gene is crucial for the regulation of insect $\mathrm{JH}$ biosynthesis [49]. Moreover, knockdown of JHAOM in the larval stage of $T$. castaneum by RNAi leads to precocious larval metamorphosis, proving $J H A O M$ is required for $\mathrm{JH}$ biosynthesis to maintain larval status [52]. Interestingly, the three enzyme genes that are involved in $\mathrm{JH}$ degradation pathway were significantly decreased, and the possible reason might be that the down-regulated $\mathrm{JH}$ biosynthetic pathway could result in lower $\mathrm{JH}$ titter, which was accompanied with the down-regulated $\mathrm{JH}$ degradation pathway during the larval metamorphosis in B. dorsalis.

Met, $K r-h 1$ and $S R C$ are the key genes in the JH signal transduction pathway [16], and Met was regarded as a receptor for $\mathrm{JH}$, and $\mathrm{JH}$-bounded Met could interact with SRC to activate $\mathrm{Kr}-\mathrm{h} 1$ in B. mori [53]. RNAi analysis showed that knockdown of Met and $K r-h 1$ could both cause precocious metamorphosis, which directly suggested their important role in the larval-pupal transition in T. castaneum $[54,55]$. JH binding protein (JHBP) could serve as a carrier to bind the JH (JH-JHBP complex) in the hemolymph and release the hormone to target tissues [56]. As expected, the $\mathrm{JH}$ signaling and transport pathway were both significantly blocked during the pupariation in $B$. dorsalis. Therefore, according to the results described above, we speculate that $20 \mathrm{E}$ and $\mathrm{JH}$ regulate metamorphosis via the regulation of specific genes involved in biosynthesis and signaling pathways in B. dorsalis.

When $B$. dorsalis developed into the pupariation, lots of cuticular protein genes were down-regulated, whereas some serine protease genes were up-regulated, indicating protein degradation might take place actively in cuticle of $B$. dorsalis during this transformation. Namely, the cuticle proteins were broken down and the chitin was released, so that the chitin could be further degraded [18]. As the vital component of the insect cuticle, the chitin of the cuticle needs to be degraded and replaced by the newly synthesized chitin during the metamorphosis. In the current study, we found that genes encoding chitinase 2 , chitinase 5 , chitinase 8 , chitinase 10 , and chitin deacetylase might participate in the chitin degradation as their expression is significantly increased during the pupariation in $B$. dorsalis. Insect chitinases are part of family 18 glycosylhydrolases that could be detected in molting fluid and gut tissues, and regulate the digestion of chitin in the exoskeleton into chitooligosaccharides [57]. Based on the RNAi experiments in T. castaneum [57] and Nilaparvata lugens [58], silencing of 
chitinase 5 and chitinase 10 genes resulted in molting defects (insect cannot degrade the old cuticle and then were trapped in their exuviates), which blocked the pupariation. For the chitin deacetylase, the injection of dsRNAs affected the molting of larval-pupal and pupal-adult metamorphosis in T. castaneum [59].

The chitin biosynthetic pathway consists of several reactions that are catalyzed by at least eight enzymes, starting with trehalase and ending with the chitin polymer [14]. As expected, the present study showed that most of the chitin biosynthesis pathway genes increased their expression, suggesting their role in the chitin synthesis during the process of pupariation in $B$. dorsalis. Chitin synthase is located at the final step of chitin biosynthetic pathway, and based on sequence similarity and function, the insect chitin synthase could be divided into two types: chitin synthase 1 (consists of two alternative splicing variants, chitin synthase $1 \mathrm{~A}$ and $1 \mathrm{~B}$ ) and chitin synthase 2 [60]. Importantly, when silencing chitin synthase $1 \mathrm{~A}$ in $\mathrm{B}$. dorsalis by RNAi, the larvae is trapped in its old cuticle and dies without pupation, while when knocking down of chitin synthase $1 \mathrm{~B}$, there was no effect on insect development [30]. In the present study, the gene expression pattern of chitin synthase $1 \mathrm{~A}$ confirmed our previous study that synthase $1 \mathrm{~A}$ is required for chitin synthesis during the larval metamorphosis in B. dorsalis [30].

\section{Conclusions}

In this study, RNA-seq analysis was performed to elucidate the molecular mechanisms of pupariation in B. dorsalis. A higher number of genes were found to have a transcriptional profile which was significantly down-regulated during this transition. Especially the genes involved in the oxidative phosphorylation pathway, cytochrome P450s, serine protease, and cuticular proteins, decreased their expressions remarkably. The present study also confirmed that the specific genes in the $20 \mathrm{E}$ and $\mathrm{JH}$ pathways might be involved in regulating the metamorphosis of $B$. dorsalis. The gene expression of chitinase 2 , chitinase 5 , chitinase 8 , chitinase 10 , chitin deacetylase, and chitin synthase $1 \mathrm{~A}$ suggested their important role in the process of pupariation. Overall, our study provided some basic gene expression data that was helpful to explore the metamorphic mechanisms of $B$. dorsalis, and functional analysis of specific genes is needed to further uncover their possible role in pupariation of $B$. dorsalis.

\section{Additional files}

Additional file 1: Table S1. Primers used for quantitative real-time PCR (qRT-PCR). (DOCX $18 \mathrm{~kb}$ )

Additional file 2: Table S2. Data output quality and mapping rates for the examined sample of Bactrocera dorsalis. (DOCX $17 \mathrm{~kb}$ )

Additional file 3: Figure. S1. Histogram distribution of genes expression level of each sample. X-axis is FPKM value (the coordinate has been changed by logarithm for better view). Y-axis is the probability density of corresponding FPKM. (JPG $469 \mathrm{~kb}$ )

Additional file 4: Table S3. All genes FPKM value during the pupariation in Bactrocera dorsalis. (XLSX $1346 \mathrm{~kb}$ )

Additional file 5: Figure. S2. Numbers of differentially expressed genes between late wandering stage and white puparium stage (DEGs, FDR $<0.01$ and $\mid \log 2$ ratio $\geq 1)$, the up-regulated genes were represented in red dot and down-regulated genes in green dot. (JPG $236 \mathrm{~kb}$ )

Additional file 6: Figure. S3. The annotated of differentially expressed genes based on different databases. Nr (NCBI non-redundant protein sequences); Pfam (Protein family); COG (Clusters of Orthologous Groups of proteins); Swiss-Prot (A manually annotated and reviewed protein sequence database); KEGG (Kyoto Encyclopedia of Genes and Genomes); eggNOG (evolutionary genealogy of genes: Non-supervised Orthologous Groups); GO (Gene Ontology). (JPG 762 kb)

Additional file 7: Table S4. Detail information about DEGs for wandering stage and late wandering stage in Bactrocera dorsalis. (XLSX 634 kb)

Additional file 8: Table S5. Detail information about DEGs for wandering stage and white puparium stage in Bactrocera dorsalis. (XLSX 683 kb)

Additional file 9: Figure. S4. GO classification of the differentially expressed genes (DEGs) in wandering stage vs. late wandering stage. (JPG $1149 \mathrm{~kb})$

Additional file 10: Figure. S5. GO classification of the differentially expressed genes (DEGs) in late wandering stage vs. white puparium stage. (JPG $1104 \mathrm{~kb}$ )

Additional file 11: Figure. S6. COG classification of the differentially expressed genes (DEGs). (A) COG classification of DEGs in wandering stage (WS) vs. late wandering stage. (B) COG classification of DEGs in WS vs. white puparium stage. (JPG $912 \mathrm{~kb}$ )

Additional file 12: Table S6. The detail FPKM value of 20-hydroxyecdysone biosynthesis and signaling related genes during the pupariation in Bactrocera dorsalis. (XLSX 12 kb)

\section{Abbreviations}

20E: 20-hydroxyecdysone; ATP: Adenosine triphosphate; BR-C: Broad complex; CA: Corpora allata; COG: Clusters of orthologous groups of proteins; CYP: Cytochrome P450; DEGs: Differentially expressed genes;

DEGs: differentially expressed genes; FAOMT: Farnesoic acid O-methyl transferase; FD: Farnesol dehydrogenase; FDR: False discovery rate;

FPKM: Fragment per kilobase of exon model per million mapped reads; FTZF1: Fushi tarazu factor 1; G-6-P-A: Glucosamine-6-phosphate Nacetyltransferase; G-6-P-I: Glucose-6-phosphate isomerase; G-F-6-PA: Glutamine--fructose-6-phosphate aminotransferase; GO: Gene ontology; $J \mathrm{H}$ : Juvenile hormone; JH: Juvenile hormone; JHAOM: Juvenile hormone acid O-methyltransferase; JHBP: Juvenile hormone binding protein; KEGG: Kyoto encyclopedia of genes and genomes; Kr-h1: Krüppel homolog 1; LWS: Late wandering stage; Met: Methoprene-tolerant; Nr: NCBI non-redundant protein sequences; Nt: NCBI non-redundant nucleotide sequences; Pfam: Protein family; PM: Phosphoacetylglucosamine mutase; SRC: Steroid receptor coactivator; Swiss-Prot: A manually annotated and reviewed protein sequence database; UDP-N-A-P: UDP-N-acetylglucosamine pyrophosphorylase; USP: Ultraspiracle protein; WPS: White puparium stage; WS: Wandering stage

\section{Funding}

This study was supported in part by the National Key Research and Development Program (2016YFC1200600), the 111 project (B18044), the earmarked fund for Modern Agro-industry (Citrus) Technology Research System (CARS-26), the Foundation Project of Southwest University (SWU1 14049), and the Fundamental Research Funds for the Central Universities, China (2362015xk04). The funders had no input into study design, data analysis, or data interpretation.

\section{Availability of data and materials}

RNA-Seq raw sequences generated using complete genomics, and have been submitted to SRA at NCBI under the Accession PRJNA420876 (wandering stage), PRJNA420907 (late wandering stage), and PRJNA420920 
(white puparium stage). Bactrocera dorsalis genome sequence data: NCBI Assembly: ASM78921v2.

\section{Authors' contributions}

E.H.C, J.J.W, and W.D. conceived the study and participated in its design. E.H.C, Q.L.H, S.F.Y, and R.L.Y. performed experiments and analyzed the data. E.H.C wrote the manuscript, which all authors have read and approved the manuscript.

\section{Ethics approval and consent to participate}

No special permits were required to collect oriental fruit flies.

\section{Consent for publication}

Not applicable.

\section{Competing interests}

The authors declare no competing interests.

\section{Publisher's Note}

Springer Nature remains neutral with regard to jurisdictional claims in published maps and institutional affiliations.

\section{Author details}

${ }^{1}$ Key Laboratory of Entomology and Pest Control Engineering, College of Plant Protection, Southwest University, Chongqing 400715, China. ${ }^{2}$ Academy of Agricultural Sciences, Southwest University, Chongqing 400715, China. ${ }^{3}$ Department of Plants and Crops, Ghent University, 9000 Ghent, Belgium.

Received: 9 December 2017 Accepted: 13 September 2018 Published online: 21 September 2018

\section{References}

1. Clarke AR, Armstrong KF, Carmichael AE, Milne JR, Raghu S, Roderick GK, Yeates DK. Invasive phytophagous pests arising through a recent tropical evolutionary radiation: the Bactrocera dorsalis complex of fruit flies. Annu Rev Entomol. 2005;50(1):293-319.

2. Wang $H_{\text {, Jin } L}$, Peng $T$, Zhang $H$, Chen $Q$, Hua $Y$. Identification of cultivable bacteria in the intestinal tract of Bactrocera dorsalis from three different populations and determination of their attractive potential. Pest Manag Sci. 2014;70(1):80-7.

3. Shen GM, Dou W, Niu JZ, Jiang HB, Yang WJ, Jia FX, Hu F, Cong L, Wang JJ. Transcriptome analysis of the oriental fruit fly (Bactrocera dorsalis). PLoS One. 2011;6(12):e29127.

4. Jan Z, Fraenkel G. The mechanism of puparium formation in flies. J Exp Zoo Part B. 2010;179(3):315-23.

5. Nachman RJ, Strey A, Zubrzak P, Zdarek J. A comparison of the pupariation acceleration activity of pyrokinin-like peptides native to the flesh fly: which peptide represents the primary pupariation factor? Peptides. 2006;27(3):527-33.

6. Dubrovsky E. B: hormonal cross talk in insect development. Trends Endocri Met. 2005:16(1):6-11.

7. Truman JW, Riddiford LM. The origins of insect metamorphosis. Nature. 1999:401(6752):447-52.

8. Truman JW, Riddiford LM. Endocrine insights into the evolution of metamorphosis in insects. Annu Rev Entomol. 2002;47(1):467-500

9. Restifo LL, Wilson TG. A juvenile hormone agonist reveals distinct developmental pathways mediated by ecdysone-inducible broad complex transcription factors. Dev Genet. 2015;22(2):141-59.

10. Reza AM, Kanamori Y, Shinoda T, Shimura S, Mita K, Nakahara Y, Kiuchi M, Kamimura M. Hormonal control of a metamorphosis-specific transcriptional factor broad-complex in silkworm. Comp Biochem Physiol B. 2004;139(4):753-61.

11. Parthasarathy R, Tan A, Bai H, Palli SR. Transcription factor broad suppresses precocious development of adult structures during larval-pupal metamorphosis in the red flour beetle, Tribolium castaneum. Mech Develop. 2008;125(3-4):299-313.

12. Yang WJ, Xu KK, Shang F, Dou W, Wang JJ. Identification and characterization of three juvenile hormone genes from Bactrocera dorsalis (Diptera: Tephritidae). Fla Entomol. 2002;99(4):648-57.

13. Yao Q, Zhang D, Tang B, Chen J, Lu L, Zhang W. Identification of 20hydroxyecdysone late-response genes in the chitin biosynthesis pathway. PLoS One. 2010;5(11):e14058
14. Liu X, Li F, Li D, Ma E, Zhang W, Zhu KY, Zhang J. Molecular and functional analysis of UDP-N-acetylglucosamine pyrophosphorylases from the migratory locust, Locusta migratoria. PLoS One. 2013;8(8):e71970.

15. Tetreau G, Cao X, Chen YR, Muthukrishnan S, Jiang H, Blissard GW, Kanost MR, Wang P. Overview of chitin metabolism enzymes in Manduca sexta: identification, domain organization, phylogenetic analysis and gene expression. Insect Biochem Mol Biol. 2015;62(1):114-26.

16. Ou J, Deng HM, Zheng SC, Huang LH, Feng QL, Liu L. Transcriptomic analysis of developmental features of Bombyx mori wing disc during metamorphosis. BMC Genomics. 2014;15(1):820.

17. Kiely ML, Riddiford LM. Temporal programming of epidermal cell protein synthesis during the larval-pupal transformation of Manduca sexta. Dev Biol. 1985:194(6):325-35.

18. Gu J, Huang LX, Gong YJ, Zheng SC, Liu L, Huang LH, Feng QL. De novo characterization of transcriptome and gene expression dynamics in epidermis during the larval-pupal metamorphosis of common cutworm. Insect Biochem Mol Biol. 2013:43(9):794-808.

19. Chen EH, Hou QL, Wei DD, Jiang HB, Wang JJ. Phenotypes, antioxidant responses, and gene expression changes accompanying a sugar-only diet in Bactrocera dorsalis (Hendel) (Diptera: Tephritidae). BMC Evol Biol. 2017;17(1):194.

20. Chen EH, Hou QL, Wei DD, Jiang HB, Wang JJ. Phenotypic plasticity, trade-offs and gene expression changes accompanying dietary restriction and switches in Bactrocera dorsalis (Hendel) (Diptera: Tephritidae). Sci Rep. 2017;7:1988.

21. Hou QL, Jiang HB, Gui SH, Chen EH, Wei DD, Li HM, Wang JJ, Smagghe G. A role of corazonin receptor in larval-pupal transition and pupariation in the oriental fruit fly Bactrocera dorsalis (Hendel) (Diptera: Tephritidae). Front Physiol. 2017;8:77.

22. Kim D, Pertea G, Trapnell C, Pimentel H, Kelley R, Salzberg SL. TopHat2: accurate alignment of transcriptomes in the presence of insertions, deletions and gene fusions. Genome Biol. 2013;14(4):R36.

23. Florea $L$, Song $L$, Salzberg SL. Thousands of exon skipping events differentiate among splicing patterns in sixteen human tissues. F1000research. 2013:2:188.

24. Schulze SK, Kanwar R, Gölzenleuchter M, Therneau TM, Beutler AS. SERE: single-parameter quality control and sample comparison for RNA-Seq. BMC Genomics. 2012;13(1):524.

25. Wang L, Feng Z, Wang X, Zhang X. DEGseq: an R package for identifying differentially expressed genes from RNA-seq data. Bioinformatics. 2010;26(1):136-8.

26. Conesa A. Blast2GO: a comprehensive suite for functional analysis in plant genomics. Int J Plant Genomics. 2008;2008(2008):619832.

27. Kanehisa M, Araki M, Goto S, Hattori M, Hirakawa M, Itoh M, Katayama T, Kawashima S, Okuda S, Tokimatsu T. KEGG for linking genomes to life and the environment. Nucleic Acids Res. 2008;36(1):D480-4.

28. Mao X, Tao C, Olyarchuk JG, Wei L. Automated genome annotation and pathway identification using the KEGG Orthology (KO) as a controlled vocabulary. Bioinformatics. 2005:21(19):3787-93.

29. Shen GM, Jiang HB, Wang XN, Wang JJ: Evaluation of endogenous references for gene expression profiling in different tissues of the oriental fruit fly Bactrocera dorsalis(Diptera: Tephritidae). BMC Mol Biol 2010, 11(1):1-10.

30. Yang WJ, Xu KK, Cong L, Wang JJ. Identification, mRNA expression, and functional analysis of chitin synthase 1 gene and its two alternative splicing variants in oriental fruit fly, Bactrocera dorsalis. Int J Biol Sci. 2013:9(4):331-42.

31. Livak KJ, Schmittgen TD. Analysis of relative gene expression data using real-time quantitative PCR and the 2(-Delta Delta $C(T))$ method. Methods. 2012;25(4):402-8.

32. Dixon GB, Davies SW, Aglyamova GA, Meyer E, Bay LK, Matz MV. Coral reefs. Genomic determinants of coral heat tolerance across latitudes. Science. 2015;348(6242):1460-2

33. Boggs CL. Understanding insect life histories and senescence through a resource allocation lens. Funct Ecol. 2009:23(1):27-37.

34. Fischer $\mathrm{K}$, O'Brien DM, Boggs $\mathrm{CL}$. Allocation of larval and adult resources to reproduction in a fruit-feeding butterfly. Funct Ecol. 2004;18(5):656-63.

35. Min KJ, Hogan MF, Tatar M, O'Brien DM. Resource allocation to reproduction and soma in Drosophila: a stable isotope analysis of carbon from dietary sugar. J Insect Physiol. 2006:52(7):763-70.

36. Hetz SK. The role of the spiracles in gas exchange during development of Samia cynthia (Lepidoptera, Saturniidae). Comp Biochem Physiol A. 2007: 148(4):743-54

37. Kaiser A, Hartzendorf S, Wobschall A, Hetz SK Modulation of cyclic CO(2) release in response to endogenous changes of metabolism during pupal 
development of Zophobas rugipes (Coleoptera: Tenebrionidae). J Insect Physiol. 2010;56(5):502-12.

38. Merkey AB, Wong CK, Hoshizaki DK, Gibbs AG. Energetics of metamorphosis in Drosophila melanogaster. J Insect Physiol. 2011;57(10):1437-45.

39. Aguila JR, Suszko J, Gibbs AG, Hoshizaki DK. The role of larval fat cells in adult Drosophila melanogaster. J Exp Biol. 2007;210(6):956-63.

40. Chiang HC. Tactic reactions of young adults of Drosophila melanogaster. Curr Biol. 2006:16(2):279-86.

41. Wolsky A. The effect of carbon monoxide on oxygen consumption of Drosophila melanogaster pupae. Pediatrics Int. 1938;15(2):23-30.

42. Terhzaz S, Cabrero P, Brinzer RA, Halberg KA, Dow JAT, Davies SA. A novel role of Drosophila cytochrome P450-4e3 in permethrin insecticide tolerance. Insect Biochem Mol Biol. 2015;67:38-46.

43. Riveron JM, Irving H, Ndula M, Barnes KG, Ibrahim SS, Paine MJ, Wondji CS. Directionally selected cytochrome P450 alleles are driving the spread of pyrethroid resistance in the major malaria vector Anopheles funestus. Proc Natl Acad Sci. 2013;110(1):252-7.

44. Petryk A, Warren JT, Marqués G, Jarcho MP, Gilbert LI, Kahler J, Parvy JP, Li Y, Dauphin-Villemant C, O'Connor MB. Shade is the Drosophila P450 enzyme that mediates the hydroxylation of ecdysone to the steroid insect molting hormone 20-hydroxyecdysone. Proc Natl Acad Sci. 2003;100(24):13773-8.

45. Cabrera AR, Shirk PD, Evans JD, Hung K, Sims J, Alborn H, Teal PE. Three Halloween genes from the Varroa mite, Varroa destructor (Anderson \& Trueman) and their expression during reproduction. Insect Mol Biol. 2015; 24(3):277-92.

46. Sugahara R, Tanaka S, Shiotsuki T. RNAi-mediated knockdown of SPOOK reduces ecdysteroid titers and causes precocious metamorphosis in the desert locust Schistocerca gregaria. Dev Biol. 2017;429(1):71-80.

47. Wan PJ, Jia S, Li N, Fan JM, Li GQ. RNA interference depletion of the Halloween gene disembodied implies its potential application for management of planthopper Sogatella furcifera and Laodelphax striatellus. PLoS One. 2014;9(1):e86675.

48. Riddiford LM. How does juvenile hormone control insect metamorphosis and reproduction? Gen Comp Endocriol. 2012;179(3):477-84.

49. Kinjoh T, Kaneko Y, Itoyama K, Mita K, Hiruma K, Shinoda T. Control of juvenile hormone biosynthesis in Bombyx mori: cloning of the enzymes in the mevalonate pathway and assessment of their developmental expression in the corpora allata. Insect Biochem Mol Biol. 2007:37(8):808-18.

50. Mayoral JG, Nouzova M, Navare A, Noriega FG. NADP+-dependent farnesol dehydrogenase, a corpora allata enzyme involved in juvenile hormone synthesis. Proc Natl Acad Sci. 2009;106(50):21091-6.

51. Burtenshaw SM, Su PP, Zhang JR, Tobe SS, Dayton L, Bendena WG. A putative farnesoic acid O-methyltransferase (FAMeT) orthologue in Drosophila melanogaster (CG10527): relationship to juvenile hormone biosynthesis? Peptides. 2008;29(2):242-51.

52. Minakuchi C, Namiki T, Yoshiyama M, Shinoda T. RNAi-mediated knockdown of juvenile hormone acid O-methyltransferase gene causes precocious metamorphosis in the red flour beetle Tribolium castaneum. FEBS J. 2008; 275(11):2919-31.

53. Kayukawa T, Minakuchi C, Namiki T, Togawa T, Yoshiyama M, Kamimura M, Mita K, Imanishi S, Kiuchi M, Ishikawa Y. Transcriptional regulation of juvenile hormonemediated induction of Krüppel homolog 1, a repressor of insect metamorphosis. Proc Natl Acad Sci. 2012;109(29):11729-34.

54. Konopova B, Jindra M. Juvenile hormone resistance gene Methoprenetolerant controls entry into metamorphosis in the beetle Tribolium castaneum. Proc Natl Acad Sci. 2007;104(25):10488-93.

55. Minakuchi C, Namiki T, Shinoda T. Kruppel homolog 1, an early juvenile hormone-response gene downstream of Methoprene-tolerant, mediates its anti-metamorphic action in the red flour beetle Tribolium castaneum. Dev Biol. 2009;325(2):341-50.

56. Ritdachyeng E, Manaboon M, Tobe SS, Singtripop T. Molecular characterization and gene expression of juvenile hormone binding protein in the bamboo borer, Omphisa fuscidentalis. J Insect Physiol. 2012;58(11):1493-501.

57. Zhu Q, Arakane Y, Beeman RW, Kramer KJ, Muthukrishnan S. Functional specialization among insect chitinase family genes revealed by RNA interference. Proc Natl Acad Sci. 2008;105(18):6650-5.

58. Xi Y, Pan PL, Ye YX, Yu B, Xu HJ, Zhang CX. Chitinase-like gene family in the brown planthopper, Nilaparvata lugens. Insect Mol Biol. 2015;24(1):29-40.

59. Arakane Y, Dixit R, Begum K, Park Y, Specht CA, Merzendorfer H, Kramer KJ, Muthukrishnan S, Beeman RW. Analysis of functions of the chitin deacetylase gene family in Tribolium castaneum. Insect Biochem Mol Biol. 2009;39(6):355-65.

60. Kato N, Mueller CR, Fuchs JF, Wessely V, Lan Q, Christensen BM. Regulatory mechanisms of chitin biosynthesis and roles of chitin in peritrophic matrix formation in the midgut of adult Aedes aegypti. Insect Biochem Mol Biol. 2006;36(1):1-9.
Ready to submit your research? Choose BMC and benefit from:

- fast, convenient online submission

- thorough peer review by experienced researchers in your field

- rapid publication on acceptance

- support for research data, including large and complex data types

- gold Open Access which fosters wider collaboration and increased citations

- maximum visibility for your research: over $100 \mathrm{M}$ website views per year

At BMC, research is always in progress.

Learn more biomedcentral.com/submissions 